\title{
Ösophaguskarzinom: Prognoseverbesserung durch neoadjuvante Radiochemotherapie
}

\section{Stefan Knippen ${ }^{1} \cdot$ Marciana-Nona Duma ${ }^{1}$}

Angenommen: 5. November 2021 / Online publiziert: 25. November 2021 (c) Der/die Autor(en) 2021

Hintergrund Die Rolle der neoadjuvanten Radiochemotherapie in der Behandlung des Ösophaguskarzinoms war lange Zeit Gegenstand kontroverser Diskussionen. Kritikpunkte vorheriger Studien betrafen v.a. das Studiendesign und oftmals auch eine geringe Fallzahl. Die Zusammenfassung der Ergebnisse verschiedener Studien in Metaanalysen lies einen Überlebensvorteil der mit RCT vorbehandelten Patienten vermuten, möglicherweise jedoch auf Kosten gesteigerter postoperativer Komplikationen. Die Veröffentlichung der Ergebnisse der randomisierten Phase-3-CROSS-Studie aus den Jahren 2012 und 2015 war und ist noch immer in der Behandlung des lokal fortgeschrittenen Ösophaguskarzinoms als ,practice-changing“ anzusehen. Eyck et al. untermauern dies in der aktuellen Veröffentlichung mit den nun vorliegenden 10-Jahres-Daten.

Patienten und Methodik 366 Patienten wurden zwischen 2004 und 2008 randomisiert mit einer neoadjuvanten RCT (nRCT; GD 41,4 Gy, ED 1,8 Gy; 5 wöchentliche Kurse Carboplatin AUC2/Paclitaxel $50 \mathrm{mg} / \mathrm{m}^{2}$ ) vorbehandelt und anschließend reseziert oder primär operiert (S). Die Strahlentherapie wurde als 3-D-konformale Therapie mit einem kraniokaudalen Sicherheitssaum von $4 \mathrm{~cm}$ und einem radialen Saum von 1,5 cm durchgeführt. Es wurden sowohl Patienten mit Plattenepithelkarzinomen (23\%) als auch mit Adenokarzinomen $(75 \%)$ des Tumorstadiums cT1 cN1 cM0 oder cT2-3 cN0-1 cM0 an 8 niederländischen Institutionen behandelt, wobei ein Großteil der Fälle als cT3 $(84 \%$ nRCT; $78 \%$ S) und cN1 (65\% bzw. 64\%) eingestuft wur-

Originalpublikation Ben M. Eyck et al (2021) Ten-Year Outcome of Neoadjuvant Chemoradiotherapy Plus Surgery for Esophageal Cancer: The Randomized Controlled CROSS Trial. J Clin Oncol 39(18):1995-2004. https://doi.org/10.1200/JCO.20.03614

Dr. med. Stefan Knippen

stefan.knippen@med.uni-jena.de

1 Klinik für Strahlentherapie und Radioonkologie, (Direktorin: Prof. Dr. med. A. Wittig), Universitätsklinikum Jena, Bachstr. 18, 07745 Jena, Deutschland de. Die Lokalisation der Tumoren lag in einem Großteil der Fälle im distalen Ösophagus (58\% nRCT, 57\% S) und im ösophagogastralen Übergang (22\% nRCT, $26 \%$ S). Insgesamt sind beide Studienarme als gut balanciert zu bezeichnen. Die Resektion erfolgte 4-6 Wochen nach der neoadjuvanten Vorbehandlung bzw. unmittelbar nach der Randomisierung. Die Patienten wurden im ersten Jahr alle 3 Monate, im zweiten Jahr alle 6 Monate und anschließend bis zum fünften Jahr jährlich nachgesorgt. Nach Abschluss des fünften Jahrs erfolgten Patientenvorstellungen bei Symptomen. Zur Erhebung der 10-Jahres-Daten wurden die betreuenden Hausärzte kontaktiert. Die Auswertung wurde folgerichtig als Intention-to-treat-Analyse durchgeführt. Primärer Endpunkt war das Gesamtüberleben (OS), sekundäre Endpunkte die krankheitsspezifische Letalität sowie die kumulativen Inzidenzen lokoregionärer Rezidive und Fernmetastasen. Die Berechnung des Follow-ups erfolgte nach reversem Kaplan-Meier-Verfahren, das Gesamtüberleben nach Kaplan-Meier, Vergleiche erfolgten nach log-Rang. Weiterhin erfolgte die uni- und multivariate Analyse von vordefinierten Subgruppen nach dem Cox-proportional-hazardsModell.

Ergebnisse Mit Ausnahme eines Patienten liegt für alle Behandelten ein minimaler Nachbeobachtungszeitraum von 120 Monaten vor. Die Vorbehandlung mit der nRCT führt zu einer signifikanten Verbesserung des Gesamtüberlebens (HR 0,7, $p=0,004$ ). So leben im nRCT-Arm nach 10 Jahren noch $38 \%$ der Behandelten, im Arm der allein resezierten Patienten noch lediglich $25 \%$. Noch deutlicher wird der Überlebensvorteil, wenn man die Unteranalyse nach Histologie betrachtet: Nach nRCT leben nach 10 Jahren noch $46 \%$ der Patienten mit plattenepithelialen Karzinomen, nach alleiniger Op. jedoch nur $23 \%$. Beim Adenokarzinom verbessert die Vorbehandlung das Überleben um absolut $10 \%$ (nRCT $36 \%$ vs. S 26\%). Die Auswertung der krankheitsspezifischen Letalität zeigt, dass der Überlebensvorteil durch eine Reduktion der Wahrscheinlichkeit, am Ösophaguskarzinom zu versterben, bedingt ist (HR 0,6, Ri- 
siko nach 10 Jahren $47 \%$ nRCT bzw. $64 \%$ S). Die Effekte scheinen durch die verbesserte lokale Kontrolle bedingt zu sein; es entwickelten nach nRCT nur $8 \%$ ein isoliertes lokoregionäres Rezidiv bzw. $13 \%$ ein lokoregionäres Rezidiv bei gleichzeitiger Fernmetastasierung. In der Gruppe der allein operierten Patienten waren dies $18 \%$ bzw. $22 \%$. In beiden Gruppen traten etwa $85 \%$ der Rezidive innerhalb der ersten 3 Jahre auf, keines aber nach dem sechsten Jahr mehr. Die Rate an alleinigen Fernmetastasen wurde durch die Therapie nicht beeinflusst (nRCT 27\%, S 28\%). Die zeitliche Kartierung des Hazard Ratio für OS zeigt, dass der Effekt der nRCT sich innerhalb der ersten 5 Jahre auswirkt, danach ist das $\mathrm{HR} \geq 1$.

Schlussfolgerung der Autoren Die neoadjuvante RCT des operablen, lokal fortgeschrittenen Ösophaguskarzinoms oder des Karzinoms des ösophagogastralen Übergangs nach dem Konzept der CROSS-Studie führt zu einer Verbesserung des OS ohne erhöhte therapiebedingte Sterblichkeit. Der Therapieeffekt persistiert auch über 10 Jahre nach einer solchen Therapie.

\section{Kommentar}

Ösophaguskarzinome gehören unter den gastrointestinalen Neubildungen zu denjenigen mit der schlechtesten Prognose. Das 5-Jahres-Gesamtüberleben beträgt, weltweit betrachtet, lediglich 15-25\%. Bei Männern ist das Ösophaguskarzinom überhaupt die sechsthäufigste krebsbedingte Todesursache [1]. Die zwei häufigsten histologischen Subtypen, Plattenepithelkarzinome und adenosquamöse Karzinome, variieren in ihrer Häufigkeit je nach geografischer Region, wobei in unseren Breitengraden und in Nordamerika die Adenokarzinome überwiegen [2]. Die unbefriedigenden Therapieergebnisse in der Behandlung lokal fortgeschrittener Ösophaguskarzinome haben zahlreiche Studien zu neoadjuvanten und adjuvanten Therapieregimen hervorgebracht, denn ohne Vorbehandlung sind trotz adäquatem Staging R1-Resektions-Raten in mehr als $25 \%$ der Fälle zu erwarten [3].

Global betrachtet unterscheidet sich die Art des neoadjuvanten Vorgehens, begründet durch die unterschiedliche Prävalenz der histologischen Subtypen. So gibt man im asiatischen Raum mit hoher Prävalenz von Plattenepithelkarzinomen den Chemotherapien den Vorzug. Das JCOG9204-Protokoll wies hierbei die Wirksamkeit einer postoperativen Cisplatin/5-FU-Kombination nach [4], JCOG9907, publiziert 2012, die Verbesserung des Überlebens durch die neoadjuvant applizierte Chemotherapie [5], die seither im Japan den Standard darstellt. In Europa und Amerika etablierte die erste Veröffentlichung der CROSSStudie im selben Jahr die neoadjuvante Radiochemothera- pie (RCT), die ihre Wirksamkeit bei beiden histologischen Entitäten unter Beweis stellte, mit höherer Wirksamkeit jedoch bei den Plattenepithelkarzinomen. Beeindruckend waren die deutliche Verbesserung der R0-Resektionsrate (92\% vs. 69\%), die Rate an pathologischen Komplettremissionen im nRCT-Arm von 29\%, mit einer pCR-Rate von $49 \%$ bei den plattenepithelialen Karzinomen, sowie die durch die nRCT verringerte Rate an postoperativ verbliebenen Lymphknotenmetastasen. Schon nach 45 Monaten Nachbeobachtung zeigte sich eine deutliche Verbesserung des Gesamtüberlebens als auch, dass die Mehrheit der verstorbenen Patienten an ihrer lokal rezidivierten Erkrankung verstarb [6]. Aktuell kann Patienten mit pathohistologisch nachgewiesener Resterkrankung (ypT1 oder ypN1) nach der nRCT die adjuvante Immuncheckpointblockade mit Nivolumab angeboten werden, die das mediane krankheitsfreie Überleben von 11 auf 22 Monate verdoppeln kann [7] und so zu einer weiteren Prognoseverbesserung beiträgt.

Ein Kritikpunkt, dem sich die CROSS-Studie trotz der hervorragenden 10-Jahres-Daten stellen muss, ist, dass die therapiebedingten Nebenwirkungen nur in den ersten 2 Jahren dokumentiert wurden. Denn Nebenwirkungen, vor allem am kardiopulmonalen System, manifestieren sich durchaus über diesen Beobachtungszeitraum hinaus. Und ein Teil von ihnen wird bereits nach 2-3 Jahren evident [8]. Der auch nach 10 Jahren nachweisbare Überlebenseffekt der nRCT manifestiert sich vor allem in den ersten 5 Jahren, sodass eine durch die RT bedingte kardiale Letalität in größerem Maße unwahrscheinlich ist. Dies wird auch durch die Literatur bestätigt [9]. Des Weiteren ergibt sich durch die heutzutage regelhaft angewendeten IMRT-Techniken eine „En-passant“-Schonung von Herz und Lungen [10].

Die Ergebnisse der ersten CROSS-Publikation haben die Behandlungsabfolge beim lokal fortgeschrittenen Ösophaguskarzinom nachhaltig verändert. Ein Streitpunkt blieben jedoch oft Karzinome des ösophagogastralen Übergangs, basierend auf den 6 Jahre zuvor publizierten Daten des MAGIC-Trials, der neben Magenkarzinomen auch $25 \%$ Karzinome des distalen Ösophagus eingeschlossen hatte und durch eine perioperative ECF-Chemotherapie das Gesamt- und das progressionsfreie Überleben verbesserte [11]. Schon 3 Jahre später legten jedoch Daten der Essener Studie nahe, dass die nRCT bei EGJ-Tumoren der alleinigen neoadjuvanten Chemotherapie vorzuziehen sei. Aufgrund der langsamen Rekrutierung erreichte jedoch das vorzeitig geschlossene Protokoll keine statistische Signifikanz [12].

Die neoadjuvante Chemotherapie wird trotzdem in dieser Frage oft in Stellung gebracht und weiterhin wissenschaftlich untersucht, zuletzt in der randomisierten Phase-II/IIIFLOT4-Studie. Diese zeigte dabei, dass das FLOT-Protokoll dem ECF-Schema vorzuziehen ist [13]. Eine durchaus bestehende antitumorale Wirksamkeit der Chemotherapie wird jedoch im Vergleich zur nRCT mit einer deutlich hö- 
heren Toxizität erkauft. Des Weiteren müssen sich die Langzeitergebnisse des FLOT-Protokolls noch beweisen. Rational betrachtet gibt es angesichts der vorliegenden 10-JahresDaten eigentlich keinen Grund, die alleinige Chemotherapie in der Behandlung der EGJ-Karzinome einzusetzen.

\section{Fazit}

Die 2012, 2015 und nun mit aktuellen 10-Jahres-Daten veröffentlichten Ergebnisse der CROSS-Studie haben die neoadjuvante RCT beim Ösophaguskarzinom fest etabliert. Bei moderaten, therapieassoziierten Nebenwirkungen erreicht die Therapie nach dem CROSS-Schema eine deutlich verbesserte Prognose, ohne die Sterberate zu erhöhen. Der Effekt des verbesserten OS resultiert vor allem aus einer verbesserten lokoregionären Kontrolle innerhalb der ersten 5 Jahre. Der hier erreichte Überlebensvorteil bleibt mindestens 10 Jahre bestehen. CROSS ist das einzige randomisierte Protokoll für diese Erkrankungsentitäten mit einer Nachbeobachtungszeit von >10 Jahren. Die auf der Basis des CROSS-Protokolls erreichbaren Ergebnisse sollten allen Betroffenen im Sinne des „shared decision-making“ empfohlen werden.

Stefan Knippen und Marciana-Nona Duma, Jena

Funding Open Access funding enabled and organized by Projekt DEAL.

Interessenkonflikt S. Knippen und M.-N. Duma geben an, dass kein Interessenkonflikt besteht.

Open Access Dieser Artikel wird unter der Creative Commons Namensnennung 4.0 International Lizenz veröffentlicht, welche die Nutzung, Vervielfältigung, Bearbeitung, Verbreitung und Wiedergabe in jeglichem Medium und Format erlaubt, sofern Sie den/die ursprünglichen Autor(en) und die Quelle ordnungsgemäß nennen, einen Link zur Creative Commons Lizenz beifügen und angeben, ob Änderungen vorgenommen wurden.

Die in diesem Artikel enthaltenen Bilder und sonstiges Drittmaterial unterliegen ebenfalls der genannten Creative Commons Lizenz, sofern sich aus der Abbildungslegende nichts anderes ergibt. Sofern das betreffende Material nicht unter der genannten Creative Commons Lizenz steht und die betreffende Handlung nicht nach gesetzlichen Vorschriften erlaubt ist, ist für die oben aufgeführten Weiterverwendungen des Materials die Einwilligung des jeweiligen Rechteinhabers einzuholen.
Weitere Details zur Lizenz entnehmen Sie bitte der Lizenzinformation auf http://creativecommons.org/licenses/by/4.0/deed.de.

\section{Literatur}

1. Pennathur A et al (2013) Oesophageal carcinoma. Lancet 381(9864): 400-412

2. Huang FL, Yu SJ (2018) Esophageal cancer: Risk factors, genetic association, and treatment. Asian J Surg 41(3):210-215

3. Kelsen DP et al (1998) Chemotherapy followed by surgery compared with surgery alone for localized esophageal cancer. N Engl J Med 339(27): 1979-1984

4. Ando N et al (2003) Surgery plus chemotherapy compared with surgery alone for localized squamous cell carcinoma of the thoracic esophagus: a Japan Clinical Oncology Group Study_JCOG9204. J Clin Oncol 21(24):4592-4596

5. Ando $\mathrm{N}$ et al (2012) A randomized trial comparing postoperative adjuvant chemotherapy with cisplatin and 5-fluorouracil versus preoperative chemotherapy for localized advanced squamous cell carcinoma of the thoracic esophagus (JCOG9907). Ann Surg Oncol 19(1):68-74

6. van Hagen P et al (2012) Preoperative chemoradiotherapy for esophageal or junctional cancer. N Engl J Med 366(22):2074-2084

7. Kelly RJ et al (2021) Adjuvant nivolumab in resected esophageal or gastroesophageal junction cancer. N Engl J Med 384(13):1191-1203

8. Bradley JD et al (2015) Standard-dose versus high-dose conformal radiotherapy with concurrent and consolidation carboplatin plus paclitaxel with or without cetuximab for patients with stage IIIA or IIIB non-small-cell lung cancer (RTOG 0617): a randomised, twoby-two factorial phase 3 study. Lancet Oncol 16(2):187-199

9. Macomber MW et al (2018) Heart dose and outcomes in radiation treatment for esophageal cancer. Cureus 10(3):e2378

10. Wu Z et al (2014) Dosimetric benefits of IMRT and VMAT in the treatment of middle thoracic esophageal cancer: is the conformal radiotherapy still an alternative option? J Appl Clin Med Phys 15(3):93-101

11. Cunningham D et al (2006) Perioperative chemotherapy versus surgery alone for resectable gastroesophageal cancer. N Engl J Med 355(1):11-20

12. Stahl M et al (2009) Phase III comparison of preoperative chemotherapy compared with chemoradiotherapy in patients with locally advanced adenocarcinoma of the esophagogastric junction. J Clin Oncol 27(6):851-856

13. Al-Batran SE et al (2019) Perioperative chemotherapy with fluorouracil plus leucovorin, oxaliplatin, and docetaxel versus fluorouracil or capecitabine plus cisplatin and epirubicin for locally advanced, resectable gastric or gastro-oesophageal junction adenocarcinoma (FLOT4): a randomised, phase 2/3 trial. Lancet 393(10184):1948-1957 